\title{
Chapitre I \\ Le portrait royal : un art officiel au service du prince
}

Le portrait princier est un art officiel façonné par des générations de peintres travaillant au service du prince avant de passer toujours plus au service de l'État. Comme d'autres artistes de l'époque, les principaux peintres qui exécutent des portraits de Marie-Thérèse suivent, pour beaucoup d'entre eux, une formation particulière, qui les amène généralement à Vienne, et tout particulièrement à l'Académie. Artisans, de plus en plus attirés et courtisés pour certains d'entre eux par les princes et les grands, les artistes entretiennent une relation particulière avec le pouvoir, et plus particulièrement avec le pouvoir royal. Dans quelle mesure peut-on parler, pour le portrait de Marie-Thérèse, d'une activité picturale encadrée par Vienne ? Dans ce chapitre, nous souhaitons nous pencher sur le rôle traditionnel du peintre de cour ainsi que sur la montée des académies au cours de l'époque moderne. Cette analyse nous semble nécessaire afin de mieux saisir l'importance et la fonction des peintres dans le processus de création de l'image royale.

\section{Le peintre de cour à l'époque moderne}

Au moins depuis la Renaissance, les princes et les grands, qu'ils soient ecclésiastiques ou nobles, s'attachent les services de peintres de cour. Ce phénomène se fait déjà sentir sous l'Antiquité, mais il devient plus fort à la Renaissance. Au cours des XVII ${ }^{\mathrm{e}}$ et XVIII ${ }^{\mathrm{e}}$ siècles, en raison de l'attraction toujours plus forte exercée par le genre du portrait, la réalisation du portrait princier est l'une de leurs principales missions. La plupart des artistes qui exécutent des portraits de MarieThérèse pour la cour sont évoqués sous les dénominations de Hofmaler ou Kammermaler, autrement dit comme les peintres de cour et de chambre, rétribués par la Kammercassa, la caisse de la Chambre. Certains de ces peintres sont aussi évoqués comme des peintres d'Académie. Les peintres d'Académie ne sont pas systématiquement des peintres de cour mais ce sont des artistes qui ont suivi les cours de l'Académie viennoise. Ces deux groupes ne coïncident donc pas toujours.

$\mathrm{Au}$ cours des siècles, le renom et la réputation des artistes deviennent des critères de choix, certains d'entre eux accèdent même à une grande célébrité européenne. En tant que protecteurs des arts, les monarques tentent d'attirer les artistes les plus prestigieux à leur cour, comme l'atteste l'exemple de Martin 
van Meytens dans notre travail. Il arrive qu'on se prête l'artiste de cour ${ }^{11}$ entre princes et grands de ce monde, quand on ne rivalise pas pour l'attirer à sa propre cour. Le prestige de l'artiste renforce celui du prince, et inversement le prince honore, en même temps qu'il oblige, l'artiste ${ }^{12}$. S'associer les grands noms artistiques d'une époque, en matière de peinture et de portraits au XVIII ${ }^{\mathrm{e}}$ siècle, s'inscrit définitivement dans une politique de prestige et de représentation réciproque.

Avec la mise en place des institutions monarchiques et étatiques, la cour exerce une attraction toujours plus forte sur les artistes. Les artistes de cour disposent d'un salaire fixe et d'une haute position à la cour ${ }^{13}$. Les fonctions artistiques du Hofkünstler, artiste de cour, ou encore du Hofkammermaler, se répandent et s'institutionnalisent à partir du XIV ${ }^{\mathrm{e}}$ siècle dans toute l'Europe ${ }^{14}$. Nous pouvons ainsi nous appuyer sur l'analyse de Martin Warnke qui estime que les princes ont libéré en grande partie les artistes du cadre corporatif artisanal en leur permettant d'exercer leur art, notamment au sein de la cour et sous la protection du prince dont ils deviennent des familiaris. Le même processus a lieu à Vienne où le service de l'empereur libère d'un certain nombre de contraintes liées aux corporations, même si les grandes familles nobles, tout comme les grands ecclésiastiques, sont d'actifs commanditaires, parfois même plus riches que la famille impériale $\mathrm{e}^{15}$. Le relatif confort financier conféré par cette position, comme l'exemption d'impôts, est toutefois contrebalancé par des attentes plus strictes du monarque. Les missions de ces artistes dépassent leurs seules activités artistiques. Il leur incombe un certain nombre de tâches comme des voyages d'inspection ${ }^{16}$ et d'entretien des châteaux et des résidences des souverains, notamment des collections royales. Ils peuvent être chargés d'en contrôler l'aménagement intérieur ${ }^{17}$, l'exemple du peintre Anton von Maron sous MarieThérèse est révélateur à cet égard ${ }^{18}$. Comme le mentionne Theresia Hauenfels, les

11 Warnke, Hofkünstler, p. 277.

12 Pour des réflexions intéressantes sur l'artiste, voir notamment Demoris, « Le Peintre pris au piège ?".

13 Hauenfels, Visualisierung von Herrschaftsanspruch, p. 60; voir aussi Warnke, Hofkünstler, pp. 142-151, notamment pp. 148-149.

14 Warnke, Hofkünstler, pp. 146-151; Hauenfels, Visualisierung von Herrschaftsanspruch, p. 59.

15 Haupt, Das Hof- und hofbefreite Handwerk.

16 Hauenfels, Visualisierung von Herrschaftsanspruch, p. 62.

17 Warnke, Hofkünstler, p. 255: « Die Innenausstattung des Schlosses im weitesten Sinne war das Arbeitsfeld des Hofmalers ».

18 Voir chapitre II, passage sur le peintre Anton von Maron ; HHSTA AKA 355 Italienische Korrespondenz 1772-75 AKA « à Monsieur le Baron de St Odile à Rome ; Schönbrun, le 27 juillet 1772. Concernant l'arrivée de M. Maron à Vienne et ses différentes commissions [. . .] » et 
peintres sont les superviseurs de l'ensemble de l'activité artistique au service du prince pendant l'époque moderne ${ }^{19}$. Enfin il existe une grande concurrence entre peintres de cour qui ne sont pas toujours payés ponctuellement ${ }^{20}$. Ce statut ne permet pas non plus toujours une grande liberté artistique, un concept de toute façon quasi inconnu à cette époque, ni non plus très pertinent ${ }^{21}$.

Dans les ateliers des artistes, le mode de réalisation demeure longtemps artisanal : le peintre en titre, auquel l'œuvre est attribuée, même s'il ne signe pas toujours, est en réalité une sorte de chef d'équipe, qui conçoit les schémas de la représentation à exécuter et se réserve souvent les parties les plus délicates. S'il dispose d'un atelier assez important, il est souvent aidé pour réaliser des tableaux ${ }^{22}$. Il peut ainsi laisser à ses assistants ou élèves le soin de peindre les vêtements, comme les dentelles chez Martin van Meytens et les décors de la scène. Ce mode de travail que l'on retrouve dans l'atelier de Martin van Meytens $^{23}$ est déjà celui de Raphaël ou de Michel Ange qui dirigent eux-mêmes leurs ateliers.

Penchons-nous sur des exemples qui servent de référence pour notre étude afin d'analyser la relation de Marie-Thérèse avec ses peintres. Nous sommes amenés à interroger le paradigme des relations entre Alexandre et Apelle, ainsi qu'entre l'empereur Charles Quint et le Titien, en nous appuyant sur le rapport privilégié entre Marie-Thérèse et son peintre principal Martin van Meytens. Comme le suggère notamment l'historienne d'art Diane Bodart, le portrait de Charles Quint réalisé par le peintre Titien constitue une sorte de « mythe d'origine » concernant les portraits des Habsbourg ${ }^{24}$. Ce portrait peint à Bologne au début des années 1530 devient une œuvre exemplaire, si ce n'est l'œuvre fondamentale dans l'historiographie de la représentation du souverain, avant même Louis XIV et son portrait par Rigaud. Cette relation entre Charles Quint et le Titien est même comparée à l'histoire et à la relation légendaire d'Alexandre le Grand et d'Apelle ${ }^{25}$.

voir aussi la lettre adressée à Monsieur le Comte de Rosenberg à Rome, « concernant l'arrivée de M. Maron à Vienne » de la même date.

19 Hauenfels, Visualisierung von Herrschaftsanspruch, pp. 59-63, p. 62.

20 Hauenfels, Visualisierung von Herrschaftsanspruch, p. 62.

21 Hauenfels, Visualisierung von Herrschaftsanspruch, pp. 61-62.

22 Nous pensons notamment aux visages et aux mains en particulier qui sont reproduits à l'identique en série.

23 Baum, Katalog des Österreichischen Barockmuseums, vol. 2, p. 425.

24 Bodart, Pouvoirs du portrait, pp. 35-49, p. 36.

25 Bodart, Pouvoirs du portrait, p. 36 ; Gaehtgens, Hochner, «Introduction », p. 11: les auteurs parlent même de topos concernant la relation entre Alexandre et Apelle, véritable leitmotiv à l'époque moderne, représentative d'une relation idéale entre le peintre et le prince. 
Le décret impérial de 1533 qui confère au peintre Titien les titres de chevalier de l'Éperon d'or et de Comte Palatin du Latran, explique que celui-ci reçoit ces honneurs en raison de sa fidélité envers l'Empire et de ses qualités, mais surtout: " de ta science exquise de peindre et de portraiturer au vif, art dans lequel tu t'es montré à nous vraiment digne d'être appelé l'Apelle de ce siècle (hujus saeculi Apelles) ${ }^{26}$. Hissant le souverain au rang d'Alexandre le Grand, le peintre est considéré comme le fidèle serviteur et même comme le premier partisan du prince, un artisan qui contribue à forger la gloire de son monarque. L'artiste met tout son art et son savoir-faire au service du pouvoir royal.

Diane Bodart rappelle que cette relation entre Alexandre et Apelle est un modèle couramment employé aux $\mathrm{XV}^{\mathrm{e}}$ et $\mathrm{XVI} \mathrm{I}^{\mathrm{e}}$ siècles pour définir un mécénat princier particulièrement généreux envers un artiste choisi, et elle rapporte l'anecdote suivante :

De même qu'Alexandre rendait visite à Apelle dans son atelier et s'entretenait familièrement avec lui ${ }^{27}$, Charles Quint fréquentait celui de du Titien et allait jusqu'à ramasser un pinceau tombé par terre : «Sire, votre serviteur ne mérite pas un tel honneur » protestait l'artiste. « Titien est digne d'être servi par César », répondait l'empereur ${ }^{28}$.

Dans tous les cas, la relation d'Alexandre et d'Apelle, puis celle entre Charles Quint et Titien, établie en exemplum, constituent une référence et un topos littéraire, reconnus et diffusés ${ }^{29}$.

Ce paradigme n'est toutefois pas entièrement applicable au cas de MarieThérèse et de Meytens dans la mesure où beaucoup d'autres peintres ont reproduit les peintures du maître suédois. Par ailleurs, il n'est pas le seul peintre de prédilection de Marie-Thérèse. On ne peut toutefois nier que la souveraine est, elle aussi, très proche de ses peintres, tout particulièrement de Martin van Meytens et de Jean-Étienne Liotard, ainsi que d'autres portraitistes qui ont la charge de la peindre. Elle leur rend volontiers visite, n'hésitant pas à les aider et à les guider en leur envoyant des esquisses de tableaux ${ }^{30}$. D'une manière générale,

26 Bodart, Pouvoirs du portrait, p. 36. Ces citations sont répertoriées et citées par Diane Bodart à partir de l'ouvrage suivant ; Cadorin, Diploma di Carlo V imperatore a Tiziano, pp. 16-17.

27 Reinach, Textes grecs et latins, pp. 314-315, cité par Bodart, Pouvoirs du portrait, p. 41 ; Gaehtgens, Hochner, «Introduction », p. 11.

28 Bodart, Pouvoirs du portrait, p. 41. Voir aussi les documents cités par Hope, « El retrato ecuestre de Carlos V de Tiziano », pp. 59-71, pp. 70-71.

29 Bodart, Pouvoirs du portrait, pp. 41-42; Hauenfels, Visualisierung von Herrschaftsanspruch, p. 59; Warnke, Hofkünstler, p. 275; voir sur ce point les propos de Checa Cremades, « Monarchic Liturgies », pp. 102-103.

30 Lisholm, Martin van Meytens, pp. 115-131. 
les séances des souverains chez les peintres ne nous sont rapportées que rarement, et de façon extrêmement lacunaire. Certaines indications laissent toutefois supposer que les moments pendant et après le déjeuner sont consacrés aux séances de pose et sont donc dédiés aux peintres ${ }^{31}$.

Une anecdote mentionnée au sujet du peintre Hubert Maurer, peintre actif durant les quinze dernières années du règne de Marie-Thérèse, par un de ses élèves est intéressante. Nous reviendrons sur cet artiste au cours de cette première partie consacrée aux peintres, car Hubert Maurer a réalisé des portraits de Marie-Thérèse :

Le prince Kaunitz avait commandé un portrait de Sa Majesté l'Impératrice pour l'envoyer à la cour russe impériale de Saint Petersbourg. L'impératrice acquiesça à cette demande et posa pour Maurer. Comme il manquait une table comme support pour sa Majesté, l'auguste impératrice aida elle-même l'artiste à porter une table de la chambre la plus proche ${ }^{32}$.

Marie-Thérèse apparaît ainsi très proche de ses artistes. Cette anecdote, peutêtre embellie, remet en cause, l'espace d'un instant, la grande différence de rang entre elle et le peintre, à l'instar de Charles Quint qui servit le Titien.

Cet exemple n'est pas le seul. Dans une lettre de Neny adressée à Saint Odile, le peintre Anton von Maron apparaît comme le peintre attitré de toute la famille impériale, les proches de Marie-Thérèse suivent très attentivement son avancement : « J'espère que Mr. Maron tirera aussi Sa Majesté l'empereur, l'archiduc Maximilien et Mme l'archiduchesse Marie et peut-être les archiduchesses Marie-Anne et Elisabeth au milieu de tout cela ». Dès son arrivée, Marie-Thérèse pose pour lui :

Le lendemain de son arrivée, je l'ai présenté à leurs Majestés Impériales, aux jeunes archiduchesses et à Monseigneur l'archiduc Maximilien, il en fut tenu au mieux et nous obtinrent de l'auguste souveraine qu'Elle se laisserait tirer par notre artiste ; en conséquence, il est venu loger hier ici, et Sa Majesté s'est déjà assise ce matin pour le premier croquis ${ }^{33}$.

Cette anecdote rappelle et souligne les relations familières entretenues par Marie-Thérèse avec certains de ses peintres.

À Vienne, la désignation de Kammermaler ou de Camermahler s'est finalement imposée. Elle signifie un « peintre de chambre » dans le sens d'un artiste

30 Lisholm, Martin van Meytens, pp. 115-131.

31 Warnke, Hofkünstler, p. 273.

32 Sattler, Lebensgeschichte, p. 49.

33 HHSTA AKA 35-5 Italienische Korrespondenz 1772-1775. Lettre de Nény à monsieur le Baron de Saint Odile à Rome, Schönbrunn le 27 juillet 1772. "Concernant l'arrivée du Sieur Maron à Vienne et ses différentes commissions ». Voir aussi chapitre II sur Maron. 
rémunéré par la Hofkammer, l'administration des finances. Remarquons que dans les livres de comptes de la cour, les Camermahler sont beaucoup moins cités que les musiciens par exemple.

La plupart des peintres de Marie-Thérèse sont mentionnés dans les Kammerzahlamtsbücher, les livres de comptes de la chambre, comme des Kammermahler $^{34}$. En effet, le titre qui permet à l'artiste de progresser au sein de la cour et de la société du prince est celui de peintre ou valet de chambre qui est conféré aux artistes dans les cours de l'époque, notamment au nord de l'Europe $^{35}$. Comme nous l'avons évoqué, ce titre implique surtout des avantages officiels : le valet de chambre accède facilement au prince en sa qualité de familiaris, le cérémonial le place à ses côtés ${ }^{36}$, il reçoit plus de revenus que les autres et obtient de grands avantages fiscaux ${ }^{37}$.

$\mathrm{Au}$ cours du XVIII ${ }^{\mathrm{e}}$ siècle, ces peintres, les Camermahler ou Kammermahler, deviennent toujours plus dépendants de l'Académie viennoise, et, la plupart d'entre eux sont formés à Vienne.

\section{Les différents statuts des artistes à Vienne}

Avant la fondation de l'Académie viennoise, trois catégories d'artistes se distinguent : les peintres bourgeois de la confraternité de Saint-Luc, les hofbefreite Künstler, brevetaires, qui peuvent exercer leur art avec l'autorisation du souverain, et enfin une troisième catégorie d'artistes qui ne jouissent ni de l'un, ni de l'autre de ces statuts ${ }^{38}$. À ceci s'ajoute la spécialité de ceux qui appartiennent en tant que cives academici, citoyens académiciens, à l'association corporative de l'université de Vienne ${ }^{39}$. Un certain nombre de différences persistent au $\mathrm{XVIII}^{\mathrm{e}}$ siècle entre les artistes: les peintres de chambre par exemple ne sont pas toujours bien acceptés dans les académies ${ }^{40}$. Toutefois, suite à l'évolution de la société, les peintres suivent de plus en plus une formation académique.

34 Voir par exemple Fleischer, Das kunstgeschichtliche Material, p. 57: « dem K. K. Cammer Mahler Johann Peter Kobler », p. 94: « Dem Cammer Mahler Pencini ».

35 Warnke, Hofkünstler, pp. 148-149, p. 149.

36 Warnke, Hofkünstler, pp. 148-149, p. 149.

37 Warnke, Hofkünstler, pp. 151-159.

38 Lützow, Geschichte der Kaiserlich-Königlichen Akademie der Bildenden Künste, p. 13; Wagner, Die Geschichte der Akademie, p. 23.

39 Lützow, Geschichte der Kaiserlich-Königlichen Akademie der Bildenden Künste, p. 13; Wagner, Die Geschichte der Akademie, p. 23.

40 Mraz, Die Geschichte der Akademie, p. 99. 
Les peintres d'histoire sont habituellement considérés comme de grands artistes, possesseurs de l'art le plus noble et occupent la première place à l'Académie où ils sont les seuls à pouvoir devenir professeurs ${ }^{41}$. La plupart des Kammermaler, des peintres de chambre du prince, sont des portraitistes tandis que les autres artistes peignent principalement des animaux ${ }^{42}$. Le portrait devient un genre de plus en plus en vogue au cours du XVIII ${ }^{\mathrm{e}}$ siècle, encouragé par le grand intérêt que lui accorde le pouvoir royal et nobiliaire mais aussi, et ce de plus en plus, par les bourgeois.

Conformément à la perception de l'histoire qui évolue au cours du temps, la conception du rôle des dirigeants dans chaque société, et plus encore leur rôle dans le processus historique, change également ${ }^{43}$. Cela a d'ailleurs un impact sur la position et sur le travail du peintre, notamment du portraitiste, dont la mission principale est de tirer le portrait de ses contemporains, en premier lieu des souverains.

Les peintres font évoluer leur manière de représenter les souverains. Ils sont donc les premiers à permettre ce changement de rôle. Même si au XVIII ${ }^{\mathrm{e}}$ siècle, le genre du portrait reste un genre intermédiaire qui n'est ni aussi prestigieux ni aussi prisé que la peinture d'histoire, en tant que premier personnage « engagé dans l'histoire ", le souverain mérite cependant d'être représenté par des artistes qui, à côté d'autres œuvres et d'autres tâches, se consacrent également et même de plus en plus à la réalisation de son portrait ${ }^{44}$.

En se détachant du cadre des corporations et en s'attachant à la personne du prince, les peintres de cour se lient toujours plus fortement au pouvoir royal. Ce rapport se retrouve encore renforcé et renouvelé par la multiplication des académies au cours de l'époque moderne. Concernant principalement Vienne, ce mouvement n'épargne pas non plus les provinces de la Monarchie.

41 Mraz, Die Geschichte der Akademie, p. 99.

42 Mraz, Die Geschichte der Akademie, p. 99.

43 Historia est à l'époque moderne un terme polysémique et qui ne peut pas toujours être assimilé à nos usages du mot « histoire »; voir Pomata, Siraisi, « Introduction », p. 28 par exemple.

44 Sur cette idée, voir notamment Pommier, «Le portrait du pouvoir », p. 4. Voir aussi Rolland, "Louis Michel Van Loo et les académies au XVIII ${ }^{\mathrm{e}}$ siècle », p. 327, comme le rapporte Rolland : «Une des idées fondamentales à la peinture d'histoire, le genre le plus prestigieux dans l'Académie, était le précepte qu'un tableau devait enrichir la vie en démontrant la noblesse des personnages héroïques et en établissant des caractères dignes d'émulation ». 


\section{Les académies et le portrait princier}

Il est nécessaire de replacer la fabrication des portraits et le travail des portraitistes au XVIII ${ }^{\mathrm{e}}$ siècle dans le contexte du développement des académies au cœur des territoires européens. Sous Marie-Thérèse, les académies de Vienne et de Milan sont les deux plus grandes de la Monarchie, directement inspirées de l'Académie de Paris. Tous les peintres, ou presque, qui ont peint Marie-Thérèse sont formés à l'Académie viennoise ou dans des académies provinciales. Les peintres qui exécutent des portraits de Marie-Thérèse sont pour ainsi dire des peintres « académiciens ». Durant cette époque, l'Académie joue un rôle croissant dans la vie artistique viennoise, toujours plus dépendante de la cour, puis de l'État. C'est dans ce contexte plus large d'académisation semi-étatique qu'apparaissent les portraits de Marie-Thérèse.

Tout comme les académies italiennes et françaises, l'histoire de l'Académie de Vienne s'inscrit dans la lignée des académies de la Renaissance. Cette institution tend à prendre de la distance par rapport aux corporations et aux guildes. Elle vise aussi à rehausser les arts au même rang que les sciences, au-dessus de l'artisanat, tout en servant le prince. À ce niveau, les peintres de cour et les peintres d'académie sont comparables, les peintres de cour suivent d'ailleurs les enseignements de l'Académie.

Faisons un petit rappel sur l'origine de l'expression « académie » qui renvoie au nom d'une partie de la ville d'Athènes où le philosophe antique, Platon, enseignait la philosophie à ses élèves. Cette expression désigne tout d'abord une réunion informelle d'humanistes ${ }^{45}$. Par la suite, les académies passent progressivement sous le contrôle de l'État et des princes comme à Florence. La première académie d'art, au sens moderne du terme, est notamment fondée à Rome à la fin du XVI ${ }^{\mathrm{e}}$ siècle, c'est l'Accademia di San Luca. Réunion des principaux peintres et artistes de l'époque, elle sert à l'éducation et à la formation des élèves ${ }^{46}$. Comme beaucoup de phénomènes artistiques, ce processus part d'Italie et touche peu à peu les différents pays européens.

En France, dans le contexte de rivalité entre les artistes de cour et les corporations, l'Académie de Peinture et de Sculpture de Paris tient sa première séance en 1648. Les statuts des académies de Florence et de Rome servent de modèles ${ }^{47}$. Ceux de cette académie parisienne serviront à leur tour plus tard

45 Mayer, « Histoire de l'Académie de Bruxelles », p. 22; Pevsner, Academies of Art, p. 1; Wagner, Die Geschichte der Akademie, pp. 13-14.

46 Wagner, Die Geschichte der Akademie, pp. 15-16.

47 Wagner, Die Geschichte der Akademie, p. 17. 
d'exemple pour l'Académie viennoise mise en place par Jacob van Schuppen. Ce n'est certainement pas un hasard si l'écrit le plus ancien conservé dans les archives de l'Académie de Vienne est une liste des membres de l'Académie parisienne de $1706^{48}$.

Ces institutions sont sous la tutelle de l'État qui les soutient et qu'elles sont également censées soutenir. On remarque que l'Académie de l'époque moderne apparaît dans le cadre d'un renforcement du pouvoir royal. Les académies sont surtout des lieux au sein desquels les artistes contemplent un certain nombre de représentations, en particulier princières, qui correspondent aux conceptions politiques du pouvoir central, relayées par les élites et les pouvoirs lo$\operatorname{caux}^{49}$. L'Académie de Paris, notamment, se doit de travailler à la gloire et à la mise en scène $d u$ Roi Soleil ${ }^{50}$. L'institution est à sa manière « une instance de proposition et de contrôle » de certains codes communs, notamment en matière picturale, que les peintres exécutent afin de mettre en scène et de célébrer la personne royale ${ }^{51}$. Même si l'Académie présente une structure d'encadrement et d'uniformisation indéniable ${ }^{52}$, le mouvement académique ne peut être réduit ou limité à une action entièrement coordonnée ni même volontariste.

À Vienne, la situation ne semble pas aussi institutionnalisée qu'à Paris, même si l'Académie viennoise s'inspire de la parisienne, obéissant ainsi à une certaine surenchère et concurrence entre souverains à ce sujet. La première académie de Peter Strudel von Strudendorff (1660-1714) ${ }^{53}$, un Tyrolien du Sud, et l'un des artistes préférés de la cour impériale, n'est que semi-officielle ${ }^{54}$.

L’Académie des Beaux-Arts de Vienne est en effet issue de l'initiative privée de Peter Strudel et du soutien de la cour impériale, de l'empereur Joseph $\mathrm{I}^{\mathrm{er}}$ surtout $^{55}$. Des mesures sont prises dès 1692 afin de resserrer officiellement les liens entre cet établissement et la cour. L'institution est alors transformée en kaiserliche

48 Wagner, Die Geschichte der Akademie, p. 17; voir aussi Schreiden, « Jacques van Schuppen », pp. 17-18.

49 Roeck, Das historische Auge, p. 91.

50 Sabatier, «La gloire du roi », pp. 528-529 ; voir aussi Pommier, Théories du portrait, p. 223. 51 Sabatier, «La gloire du roi », p. 530 ; voir aussi Sabatier, « Les rois de représentation ».

52 Rolland, "Louis Michel Van Loo et les académies au XVIII ${ }^{\mathrm{e}}$ siècle », p. 322; en s'appuyant sur l'exemple espagnol, Rolland affirme que l'Académie devait « favoriser les artistes locaux » en les formant et en évitant d'en importer.

53 Voir à ce sujet l'ouvrage de Manfred Koller, Die Brüder Strudel.

54 Wagner, Die Geschichte der Akademie, pp. 18-19. Bien que ses débuts restent dans l'ombre en raison du manque de sources, sa fondation renvoie à l'année 1692.

55 Il est possible que l'enseignement ne commençât en fait qu'en 1705, au début du règne de Joseph I ${ }^{\mathrm{er}}$. Son biographe Eucharius Gottlieb Rinck le célèbre en tout cas comme le fondateur de l'Académie ; voir Wagner, Die Geschichte der Akademie, pp. 19-20. 
Academie ou Hof-Academie, c'est-à-dire en Académie impériale ou Académie de cour. Cependant, la fondation de l'établissement n'est officialisée par aucun document. Toujours privée, l'Académie est désormais sous protection impériale. Elle perçoit, à l'occasion, des subventions. C'est sous cette forme que perdure l'académie de Strudel jusqu'à la mort de celui-ci en 1714, date à laquelle elle est dissoute ${ }^{56}$.

En 1726, le peintre Jacob van Schuppen (1670-1751) obtient l'autorisation de Charles VI de réorganiser l'institution semi-officielle de Strudel dont il devient directeur et qui est alors rémunérée par la $\operatorname{cour}^{57}$. L'empereur reconnaît la validité de l'académie, il nomme Schuppen Préfet et Directeur de l'Académie et le soumet à l'Obersthofmeister, au grand maître de cour. Schuppen obtient la permission de s'inspirer de modèles parisiens et de poursuivre son travail avec son atelier, en studio, à la manière italienne, ce que l'on retrouve notamment par la suite chez Martin van Meytens ${ }^{58}$. Ses membres peuvent porter le titre de peintre ou de sculpteur impérial, ce qui est un grand honneur qui n'est pas accordé à tous les artistes.

Les informations dont nous disposons sur l'installation de l'Académie à l'époque de Léopold $\mathrm{I}^{\mathrm{er}}$ et de Joseph $\mathrm{I}^{\mathrm{er}}$ sont très éparses. On sait par contre plus de choses au sujet de la nouvelle fondation de l'Académie sous Charles VI. Indéniablement, l'influence de l'Académie de Paris sur celle de Vienne se fait sentir très fortement avec l'arrivée de Jacob van Schuppen. Si l'institutionnalisation de l'image royale est un sujet dominant pour les plus grands conseillers royaux, tout particulièrement en France, un processus similaire se répète à peu de choses près à Vienne sous Charles VI, puis sous Marie-Thérèse ${ }^{59}$.

\section{L’Académie de Vienne et les académies dans les provinces de la Monarchie : lieu de formation des portraitistes de Marie-Thérèse}

Marie-Thérèse et les peintres qui lui sont attachés à la cour de Vienne sont au cœur de la production de portraits. L'Académie de Vienne concentre en grande partie la réalisation du portrait royal que cela soit d'une manière directe, par la

56 Wagner, Die Geschichte der Akademie, p. 21.

57 Wagner, Die Geschichte der Akademie, pp. 21-22.

58 Schreiden, « Jacques van Schuppen », pp. 15-28; voir aussi Garas, « Über Meister und Vorgänger Maulbertschs », pp. 22-29.

59 Les statuts rédigés pour Vienne par Schuppen sont calqués sur ceux de l'Académie parisienne; voir Lützow, Geschichte der Kaiserlich-Königlichen Akademie der Bildenden Künste, p. 14. 
commande et la réalisation de portraits par des membres de l'Académie, mais aussi de façon indirecte en formant des peintres originaires des différentes provinces de la Monarchie. L’Académie demeure en effet le principal lieu d'apprentissage des artistes de l'époque, et donc des portraitistes de Marie-Thérèse quel que soit leur lieu d'activité, à l'instar du peintre hongrois Daniel Schmidelli qui exerce pour un public hongrois mais qui a été auparavant l'élève de l'Académie de Vienne.

La concurrence de la France, toujours plus puissante, d'un point de vue aussi bien culturel que politique, contribue à accroître l'intérêt des Habsbourg, en particulier de Charles VI, puis de sa fille Marie-Thérèse et de ses conseillers, pour l'institution académique. Il s'agit de mettre en scène une grande puissance habsbourgeoise, dans le contexte de la perte de la couronne espagnole au profit des Bourbons, en développant une composition artistique spécifique qui doit manifester cette position éminente.

L'Académie a-t-elle une fonction régulatrice dans la formation de portraits officiels de Marie-Thérèse ? Les peintres qui y sont formés livrent en effet une vision stéréotypée et facilement reconnaissable de la souveraine, surtout pour la postérité : que l'on songe aux nombreux portraits réalisés par l'artiste principal Martin van Meytens qui se trouvent aujourd'hui encore dans nos manuels d'histoire, en particulier l'image de Marie-Thérèse à côté de la couronne de Hongrie, réalisée en 1759, par Meytens pour les nouveaux bâtiments de l'Académie de Vienne. Il est cependant difficile d'apprécier l'influence véritable de l'Académie. Un certain nombre de normes, de schémas peuvent aussi être respectés par tous les peintres, non seulement par les artistes formés à l'Académie, car ces types de portraits correspondent à des motifs hérités et influencés par l'époque.

Après la mort de Schuppen en 1751, Martin van Meytens, portraitiste principal de Marie-Thérèse, obtient le poste de directeur de l'Académie en 1759 qu'il conserve lui aussi pour le restant de sa vie jusqu'en $1770^{60}$. Les deux directeurs ont pour point commun de venir de familles originaires des Pays-Bas, ils ont par ailleurs été à l'Académie Royale de Paris où ils ont principalement exercé leur métier et leur art de portraitiste ${ }^{61}$. Il est remarquable, si l'on se rapporte à la hiérarchie des genres qui pendant longtemps privilégie les peintres d'histoire, que la direction de l'Académie soit confiée à des peintres qui sont avant

60 Wagner, Die Geschichte der Akademie, p. 27, Wagner explique ainsi la nomination de Meytens : « Seine gründliche Kenntnis der Kunst, die er sich auf ausgedehnten Reisen in Holland, England, Frankreich und Italien erworben hatte, seine Welt- und Menschenkenntnis sowie angenehme persönlichen Eigenschaften ließen ihn für diese Stelle als sehr geeignet erscheinen ».

61 Dachs, « Der Geschmackswandel », p. 267; Garas, « Über Meister und Vorgänger Maulbertschs », pp. 22-23. 
tout des portraitistes ${ }^{62}$. Comme nous l'avons évoqué, une évolution s'opère au cours du XVIII ${ }^{\mathrm{e}}$ siècle, le genre du portrait commence à dominer.

\section{Les académies dans les provinces de la Monarchie}

Même dans les provinces éloignées du centre viennois, les académies jouent un rôle toujours plus important dans la formation des peintres et au cœur de l'activité artistique de l'époque, surtout au cours de la seconde moitié du XVIII ${ }^{\mathrm{e}}$ siècle. La création d'académies a principalement lieu à Vienne mais aussi dans des provinces plus excentrées qui ne font pas partie des pays héréditaires ; mentionnons les provinces italiennes ainsi que celles des Pays-Bas autrichiens. Une tradition artistique très ancienne et encore foisonnante au XVIII ${ }^{\mathrm{e}}$ siècle, peut expliquer le développement académique en ces lieux. Il est possible d'y lire aussi une manière détournée pour ces provinces de témoigner d'une certaine indépendance par rapport à Vienne en rivalisant avec la capitale viennoise comme avec son académie, d'un point de vue artistique du moins. Toutefois, ces académies provinciales sont encouragées par Marie-Thérèse. Le pouvoir central viennois s'infiltre ainsi par l'entremise des académies dans ces provinces riches et stratégiques. Il est surtout plus probable d'y voir l'intérêt porté par la souveraine à tous les domaines et les secteurs qui concernent de près ou de loin l'art et la culture.

En Italie, vers 1600, seules les villes de Florence et de Rome, en raison de leur ancienneté et de leurs traditions, disposent de systèmes académiques assez importants et respectés pour concurrencer et s'imposer face au système traditionnel des corporations ${ }^{63}$. Par la suite, Milan imite le modèle romain en créant une académie. Passée sous l'administration des Habsbourg d'Autriche au début du XVIII ${ }^{\mathrm{e}}$ siècle, après la guerre de Succession d'Espagne, la capitale lombarde est indéniablement un centre de pouvoir politique, culturel et artistique. Les échanges économiques et culturels sont forts entre les grandes académies de la Monarchie comme Vienne et Milan. Semblable en de nombreux points à l'Académie de Vienne, dont elle est devenue un relais et une protégée au sein de l'espace méridional de la Monarchie, l'Académie de Milan s'occupe surtout de fournir une formation sérieuse à ses étudiants, respectant en cela un des points essentiels de toute académie.

62 Voir aussi un article intéressant sur le sujet ; Rolland, « Louis Michel Van Loo et les académies au XVIII ${ }^{\mathrm{e}}$ siècle », pp. 317-340.

63 Pevsner, Academies of Art, pp. 67-71. 


\section{Le développement d'académies dans les Pays-Bas autrichiens}

Les Pays-Bas méridionaux forment une autre province acquise par les Habsbourg d'Autriche au début du XVIII ${ }^{\mathrm{e}}$ siècle, à la suite de la guerre de Succession d'Espagne et de la perte définitive des territoires espagnols par la dynastie des Habsbourg, et qui vont devenir les Pays-Bas autrichiens. Dans cette région excentrée de Vienne, les académies se développent aussi aux XVII ${ }^{\mathrm{e}}$ et XVIII ${ }^{\mathrm{e}}$ siècles. Plusieurs peintres de notre corpus de portraits peuvent être rattachés à une académie flamande. Au premier rang desquels figure le directeur de l'Académie de Bruges, Matthias de Visch. Les Pays-Bas disposent indiscutablement d'une tradition picturale et artistique propre très riche et encore très vivante au $\mathrm{XVIII}^{\mathrm{e}}$ siècle. Il n'est donc pas étonnant qu'un nombre relativement significatif de portraitistes ayant exécuté des tableaux de Marie-Thérèse soient issus de cette province.

Le gouverneur de la région, Charles de Lorraine, beau-frère de MarieThérèse, soutient les artistes de manière individuelle, à titre privé, formant une sorte de cour qui n'est pas forcément si dissemblable de la cour viennoise ${ }^{64}$. Il joue un rôle très important en matière culturelle et artistique. Par la suite, l'arrivée de Cobenzl marque fortement la politique artistique de son époque lors de sa nomination comme ministre plénipotentiaire en $1753^{65}$. Ce dernier, amateur d'art comme Kaunitz ou d'autres hommes politiques et conseillers de Marie-Thérèse, donne de fortes impulsions " aux mesures concrètes du mécénat officiel » ${ }^{66}$.

En 1763, Charles de Lorraine accorde même son soutien aussi bien à l'Académie de Bruxelles qu'à celle d'Anvers. « Le nombre de projets et de mémoires attestent de l'intérêt porté par le pouvoir à la Réforme de l'Académie ${ }^{67}$. Mais penchons-nous sur l'Académie de Bruges particulièrement intéressante pour notre propos grâce à son directeur, Matthias de Visch, qui réalise plusieurs portraits de Marie-Thérèse de notre corpus (P 123, Figure 10 et P 124, Figure 11). Peintre d'histoire, de sujets religieux et de portraits à Bruges, Matthias de Visch (1701-1765) est lui-même élève à l'Académie des Beaux-Arts de Bruges. Il a pu alors entreprendre un voyage qui l'a mené à Paris, à Parme, à Rome et à Venise ${ }^{68}$. Ce peintre qui exécute deux portraits de Marie-Thérèse pour la ville de

64 Galand, «Charles de Lorraine et la vie artistique ».

65 Galand, Charles de Lorraine, p. 28.

66 Galand, Charles de Lorraine, p. 28.

67 Mayer, « Histoire de l'Académie de Bruxelles », p. 26.

68 Fornari, Jacobs, « De Visch »; Meulemeester, « Mathias de Visch », pp. 291-292. 
Bruges est représentatif de l'importance jouée par l'institution académique dans des provinces éloignées du centre viennois. L’Académie de Bruges est influencée par l'Académie parisienne tout autant que par celle de Vienne ${ }^{69}$.

L'Académie brugeoise exerce en effet une fonction essentielle à cette époque $^{70}$. Le premier lauréat de cette Académie en 1721 n'est autre que le peintre Matthias de Visch ${ }^{71}$. En 1738, De Visch ouvre de nouveau les portes de l'Académie de Bruges qui avait été fermée, faute de moyens ${ }^{72}$. Il devient administrateur, premier professeur et directeur de l'Académie de Dessin, de Peinture, de Sculpture et d'Architecture de Bruges. Après avoir fondé une école de dessin privée, De Visch reçoit une salle de l'hôtel de ville pour y pratiquer son enseignement dès $1739^{73}$. Deux portraits de Marie-Thérèse sont d'ailleurs encore suspendus dans cet hôtel de ville (P 123, Figure 10 et P 124, Figure 11).

Si la plupart des académies procèdent des intentions royales centralisatrices, l'Académie de Bruges, comme celle de Bruxelles, doit son soutien aux corporations, ainsi qu'aux communes très puissantes et respectées dans ces provinces ${ }^{74}$. Il est intéressant de noter que le pouvoir royal viennois ne fait, dans certains cas, que renforcer l'action communale et locale des élites des provinces.

Sous le règne de Marie-Thérèse, les peintres d'académie deviennent de plus en plus des peintres de cour, même si les guerres de Succession d'Autriche ont indéniablement freiné l'activité académique ${ }^{75}$. Il n'est en effet pas anodin que le portraitiste le plus connu de Marie-Thérèse, Martin van Meytens, soit nommé directeur de l'Académie de Vienne.

Bien que leurs objectifs diffèrent, ateliers et académies, échangent et s'influencent mutuellement, tout particulièrement avec la nomination de Martin van Meytens comme directeur de l'Académie viennoise à la fin des années 1750 .

Même après la mort de Meytens, lorsque le chancelier d'État Kaunitz nommé protecteur de l'Académie en 1772 s'occupe personnellement des affaires de l'institution, les portraits des membres de la famille impériale, notamment les portraits de famille, sont toujours réalisés par de nombreux artistes. Une lettre datant de

69 Dendooven, «Les collections d'artistes à Bruges », p. 109, l'auteur affirme que Bruges était particulièrement bien « représentée au sein de l'Académie parisienne ».

70 Voir à ce sujet Dendooven, « Les collections d'artistes à Bruges », p. 109.

71 Dendooven, «Les collections d'artistes à Bruges », p. 108.

72 Dendooven, «Les collections d'artistes à Bruges », p. 108.

73 Mayer, « Histoire de l'Académie de Bruxelles », p. 23.

74 Mayer, « Histoire de l'Académie de Bruxelles », p. 23.

75 Walter Wagner parle d'une mauvaise époque pour l'Académie de Vienne sous la direction de Martin van Meytens, le nombre d'élèves aurait diminué et l'enseignement n'aurait pas été à la hauteur ; voir Wagner, Die Geschichte der Akademie, p. 28. 
1775, écrite par Kaunitz à Marie-Thérèse, évoque des esquisses de grands portraits de famille faites par dix personnes différentes. Il pourrait bien s'agir des différents spécialistes d'un atelier, chargés chacun d'une partie du portrait :

Madame ! J'ai l'honneur d'envoyer à Votre Majesté des Esquisses pour les grands portraits de famille qu'elle m'a commandés. Ils sont faits par 10 personnes différentes, et Votre Majesté jugera mieux que personne du degré de leur valeur ${ }^{76}$.

Entre 1759, date de la nomination de Martin van Meytens à la tête de l'Académie, et 1770 , lors de sa mort, l'Académie semble se confondre avec l'atelier de celui-ci. Le peintre est alors à la tête d'un atelier d'artistes qui réalise des portraits de Marie-Thérèse destinés aux élites, à leur propre demande, ou offerts par la cour ${ }^{77}$. Le neveu, compatriote et élève de Meytens, Sophonias de Derichs $^{78}$, est probablement le membre le plus connu de cet atelier qu'il dirige avec son oncle. Cette association permet à Meytens de produire une quantité impressionnante de portraits individuels et de groupes avec la souveraine, sa famille et la cour de Vienne. La plupart des portraits de Marie-Thérèse leur sont attribués à tous deux, même s'ils ne sont ni datés ni signés, chose habituelle à l'époque $^{79}$. En sa qualité de directeur de l'Académie, il joue un rôle décisif et permet de relier la cour et l'Académie. En devenant une référence par excellence, il contribue à transformer le portrait de Marie-Thérèse qui passe d'une simple représentation de cour à une représentation académique, uniformisée et en apparence normalisée.

76 AVA Unterricht StHK Teil 1757 Wien, Akademie der bild. Künste; 1773-1783; fo. 21, 17 Mars 1775, lettre de Kaunitz à Marie-Thérèse.

77 « Leben Herrn Martin von Meytens, dessen Tod wir im 16ten Stück der Zeitung angezeigt haben ", in Kunstzeitung der Kaiserlichen Akademie zu Augsburg, (1770), cité d'après Lisholm, Martin van Meytens, p. 122: « Er verfertigte Gemälde, über Gemälde; denn jede Nation gieng ihn um Werke seiner Hand an; und zum Glück arbeitete er nicht langsam ». À ce sujet, voir la remarque de Baum, Katalog des Österreichischen Barockmuseums, vol. 2, p. 425: «Die große Menge von Bestellungen konnte nur mit Hilfe eines Werkstattbetriebes bewältigt werden ».

78 Björk, « De Derichs ».

79 Baden (éd.), Briefe über die Kunst, p. 221 : «Il n'a mis son nom sur les Tableaux ou Portraits que fort rarement lorsqu'on l'a absolument exigé ». Voir aussi Baum, Katalog des Österreichischen Barockmuseums, vol. 2, p. 425. Sur l'atelier et les élèves de Meytens, voir Lechner, « Martin van Meytens ", pp. 16-19; Lisholm, Martin van Meytens, pp. 80-84. 


\section{Réformes académiques autour de $\mathbf{1 7 7 0}$}

Pendant la période de réformes de l'académie viennoise dans les années 1770, durant la dernière décennie de règne de la souveraine, de grands peintres de Marie-Thérèse comme Anton von Maron ainsi que des hommes politiques tels le chancelier d'État Kaunitz s'impliquent fortement dans ce mouvement de réforme artistique et académique ${ }^{80}$. La réforme de l'éducation académique s'appuie principalement sur le modèle romain comme en témoigne la recommandation par le peintre Maron de l'ouvrage de son confrère Anton Raphael Mengs, Gedanken über die Schönheit und über den Geschmack in der Malerei. Ce livre théorise un certain nombre des évolutions qui étaient en cours en matière artistique ${ }^{81}$. Entre 1772 et 1783, la Monarchie des Habsbourg finance aussi des séjours de boursiers à Rome pour huit élèves de l'Académie de Vienne et pour trois élèves de l'Académie de Milan. Le premier groupe de boursiers viennois, avec entre autres les artistes Christian Sambach, Hubert Maurer et Johannes Tusch, revient à Vienne au bout de quatre années, en juin $1776^{82}$; les deux derniers ont peint plusieurs portraits de Marie-Thérèse veuve. Toute une génération de portraitistes de Marie-Thérèse est très marquée par les séjours romains et par l'influence plus ou moins directe d'hommes d'État comme Kaunitz et Sonnenfels sur la formation des artistes.

Invité lors de la remise de prix solennelle le 23 septembre 1768 à l'Académie de gravure sur cuivre de Vienne, le juriste et théoricien Joseph Sonnenfels tient un discours $A u$ sujet du mérite du portraitiste ${ }^{83}$. Dans ce discours, il décrit l'importance de l'art pictural, en particulier du portrait, en évoquant les exemples antiques. L'intérêt de Sonnnenfels pour les théories artistiques correspond aux inclinations personnelles du comte de Kaunitz, chancelier d'État de MarieThérèse. Cet intérêt est également lié aux écrits du théoricien d'art Johann Joachim Winckelmann concernant l'histoire de l'art et sa théorisation ${ }^{84}$. Ces propos nous permettent de replacer les tableaux dans l'esprit d'une époque

80 Kroupa, « Fürst Wenzel Anton Kaunitz-Rietberg », pp. 369-371; Szabo, Kaunitz and Enlightened Absolutism, pp. 200-204.

81 Mengs, Gedanken über die Schönheit. Concernant la recommandation de Maron, qui était le beau-frère de l'auteur, voir Lützow, Geschichte der Kaiserlich-Königlichen Akademie der Bildenden Künste, p. 54.

82 Fleischer, Das kunstgeschichtliche Material, pp. 40-41; voir aussi Wagner, Die Geschichte der Akademie, pp. 45-46.

83 Sonnenfels, Von dem Verdienste des Portraitmalers; voir aussi Karstens, Lehrer - Schriftsteller-Staatsreformer, p. 162.

84 Szabo, Kaunitz and Enlightened Absolutism, pp. 24-25. 
où l'on observe la mainmise progressive de l'État et des hommes politiques sur l'activité artistique. L'activité du peintre s'institutionnalise progressivement, notamment sa formation artistique.

La politique accorde une place importante à l'art comme au portrait dans la formation de l'État ${ }^{85}$. Ainsi, pendant de nombreuses années, Joseph Sonnenfels sert les Habsbourg, et en particulier Marie-Thérèse. Morave, issu d'une famille juive convertie, personnalité marquante des Lumières autrichiennes, Sonnenfels est nommé en 1763 à la chaire des sciences camérales de l'université de Vienne $^{86}$, il devient ensuite secrétaire permanent de l'Académie de gravure. Il est particulièrement intéressant et significatif qu'un tel personnage, un écrivain et un responsable politique des Lumières, ait écrit des ouvrages sur le mérite du métier de portraitiste. Son traité principal est exposé dans un discours tenu en janvier 1768 lors d'une visite de Kaunitz pour une première remise de prix. Le discours s'intitule alors : Ermunterung zur Lektüre an junge Künstler, Encouragement à la lecture destiné aux jeunes artistes ${ }^{87}$. Si l'on se place du point de vue de Sonnenfels, il est essentiel que les souverains soutiennent les arts. De même, les grandes familles de la Monarchie sont également incitées et invitées à faire exécuter leurs portraits ${ }^{88}$.

Le rôle du chancelier d'État, le prince Wenzel Anton Kaunitz, est peut-être plus important encore que celui de Sonnenfels, il est primordial dans la vie artistique autrichienne ${ }^{89}$. C'est dans un rapport concernant le plan de réunification des écoles d'artistes, rendu le 25 mai 1770, que Kaunitz exprime ses considérations sur la signification de l'art pour le développement de l'État ${ }^{90}$. Kaunitz souhaite créer une Académie des Beaux-Arts et des Sciences (Akademie der Schönen Künste und schönen Wissenschaften), soulignant ainsi le lien entre l'art et les sciences, l'art de la peinture et du portrait permettant de soutenir le bien de l’État.

85 Karstens, Lehrer - Schriftsteller - Staatsreformer, pp. 161-162.

86 Reinalter, «Joseph von Sonnenfels », pp. 3-4. Sur cette question de l'influence de Kaunitz et de Sonnenfels sur l'art, voir aussi Fidler, «Wandel der Themen », p. 37; Kaufmann, Court, Cloister, and City, p. 443.

87 Sonnenfels, Gesammelte Schriften, vol. 8, pp. 275-296.

88 Sonnenfels, Gesammelte Schriften, vol. 8, pp. 370-372, 400-401.

89 AVA Unterricht StHK Teil 175 7, Kaunitz s'adresse même directement à Marie-Thérèse pour lui évoquer le sort des peintres ainsi que la gestion des commandes de tableaux, portraits y compris. Voir archives citées, Allgemeines Verwaltunsgarchiv, Unterricht und Kultus, 75 Studien-Hofkommission, Sign.: 15 Akademie AVA; 175 7; Wien Akademie der bild. Künste, 1774-1783, fo. 21.

90 Wagner, Die Geschichte der Akademie, pp. 37-39. 
La protection directe du chancelier d'État est un signe important d'étatisation de l'activité artistique. Il n'est donc pas étonnant que l'on retrouve aujourd'hui encore des rapports envoyés par Kaunitz à la souveraine où il lui recommande certains peintres comme Anton von Maron, Johannes Tusch ou Hubert Maurer. Kaunitz lui rapporte également tout ce qui se déroule en termes d'activité artistique ${ }^{91}$. Les hommes d'État, à l'instar des princes, s'intéressent en effet à l'art, tout particulièrement à l'art du portrait.

Les académies de peinture et de gravure ${ }^{92}$ sont réunies en 1772 par Kaunitz, soit deux ans après la mort de Meytens. Il est possible d'y voir un nouveau positionnement de la politique académique après la mort d'un des derniers représentants de la vieille école. La souveraine transmet à l'un de ses nouveaux peintres, ou du moins à celui qui dispose d'un certain renom depuis la mort de Meytens, et qui travaille avec Kaunitz, le peintre Anton von Maron ${ }^{93}$, le projet d'unification des académies. En 1772, Kaunitz annonce en effet la réunion des académies en une Kaiserlich-Königlich vereinigte Akademie der bildenden Künste, l'Académie impériale et royale des Beaux-Arts, qui comprend alors les écoles de dessin, de peinture, de sculpture, d'architecture et de gravure ${ }^{94}$.

Die Kaiserliche-Königliche-Hof-Academie für Mahlerey, Bildhauerey und Baukunst, l'Académie de cour royale et impériale pour la peinture, la sculpture et l'architecture, est dès lors intégrée comme partie principale dans l'établissement élargi des freye, vereinigte Academie der bildenden Künste, l'Académie réunie libre des arts plastiques, également désignée sous le nom freye Academie der vereinigten bildenden Künste. Kaunitz, soutenu par Maron, met alors en place des réformes importantes en matière d'enseignement et d'organisation, qui visent à influencer le style et l'art de l'Académie vers l'art romain. Plus qu'une fonction honorifique, la position de Kaunitz comme protecteur lui permet un véritable engagement et une vraie influence dans l'institution ${ }^{95}$.

91 AVA Unterricht StHK Teil 1 A 757 Wien Akademie der bildenden Künste, fo. 21.

92 En plus de l'institution dirigée par Meytens, une Académie de gravure se développe sous la direction de Jacob Schmutzer (1733-1811), Cette fondation est due à l'initiative de la cour ellemême ; Heinz, «Bemerkungen », p. 278 ; voir aussi Kaufmann, Court, Cloister, and City, p. 412. C'est pour la période entre 1766 et 1777 que nous possédons le plus d'informations au sujet de la nouvelle institution, dont Kaunitz lui-même est le protecteur. Marie-Thérèse accorde d'ailleurs à la Kupferstecherakademie un statut écrit, lui donnant force de loi.

93 Schmittmann, Anton von Maron, pp. 45-48.

94 Wagner, Die Geschichte der Akademie, pp. 37-43.

95 Szabo, Kaunitz and Enlightened Absolutism, p. 204; voir aussi Kroupa, « Fürst Wenzel Anton Kaunitz-Rietberg », p. 371. 
L'Académie, sous Martin van Meytens, avait été avant tout une école de cour, tandis que celle de Kaunitz est davantage une école publique, au service de l'État. Les réformes entreprises au sein de l'Académie ont lieu juste après la mort de Meytens, manière probablement de respecter le vieil artiste et ami de tous, un des derniers représentants d'une structure d'un autre temps, à qui on a voulu laisser jusqu'à sa mort le soin de diriger l'ancienne organisation académique.

L'influence de l'écrivain Franz Christoph von Scheyb ${ }^{96}$, autre théoricien écrivant sur le genre du portrait, sur la réforme académique de 1772 ne peut être non plus oubliée. Scheyb exprime ses réflexions artistiques dans une œuvre qui se compose de deux volumes : Köremons Natur und Kunst in Gemälden, Bildhauereyen, Gebäuden und Kupferstichen [. . .], La nature et l'art de Köremon et l'art dans les tableaux, les sculptures, les bâtiments et les gravures, publiés à Leipzig en 1770 et Orestrio-von den drey Künsten der Zeichnung, l'Orestrio-au sujet des trois arts du dessin, édité à Vienne en 1774. Les beaux-arts, dont la peinture fait partie intégrante, permettent de documenter les actes des générations passées pour celles à venir. Ce sont des arts utiles. Ces écrits de Scheyb sont tous deux dédiés à Martin van Meytens, le grand peintre et portraitiste du règne. Scheyb apprécie en effet le peintre officiel de Marie-Thérèse. Il lui a même consacré dans ses deux œuvres un éloge particulier ${ }^{97}$ et retranscrit leur échange de réflexions dans un de ses écrits sous les pseudonymes de Köremon et d'Orestrio Zum Unterricht der Schüler und Vergnügen der Kenner, Pour l'enseignement des élèves et le plaisir des connaisseurs ${ }^{98}$.

\section{Conclusion}

$\mathrm{Au}$ cours du XVIII ${ }^{\mathrm{e}}$ siècle, le genre du portrait fait de plus en plus l'objet d'interrogations et d'analyses, témoignant de son importance grandissante. Des hommes politiques et non des moindres, tel le chancelier d'État Kaunitz ou Sonnenfels, se

96 Holzer, Franz Christoph Scheyb.

97 Übelleitner, Tugenden Maria Theresias.

98 Scheyb, Köremons, Natur und Kunst in Gemälden. Cet ouvrage comporte une dédicace et une lettre à Martin van Meytens: " An den erlauchten, gelehrten und ruhmvollen Herrn Martin van Meytens, Ihro Kaiserl. Königl. Apostol Majestät ersten Kammer-und Kabinetsmaler wie auch Allerhöchst Derosselben freyen Akademie der Malerey, Bildhauerkunst und Architektur zu Wien gevollmächtigten Director. / A l'illustre, érudit et glorieux Monsieur Martin van Meytens, le premier peintre de cabinet de chambre de sa majesté apostolique, impériale et royale, et directeur tout puissant de la plus haute académie libre de peinture, de sculpture et d'architecture à Vienne ». 
penchent sur la question de la formation des peintres, et en particulier des portraitistes. Pour eux, l'art du peintre est un art utile au service du prince mais aussi de l'État et du bien public. C'est pourquoi l'éducation des artistes doit faire l'objet d'un encadrement plus institutionnalisé de la part de l'État. Vienne n'est pas la seule ville de la Monarchie à posséder une Académie même si c'est surtout l'évolution de cette dernière qui intéresse notre propos. Les académies provinciales ne sont pas à négliger pour autant car elles forment des peintres qui sont appelés à représenter la souveraine, notamment dans les régions périphériques de la Monarchie.

Le temps des académies succède au temps des cours. On assiste à une académisation, c'est-à-dire à une institutionnalisation du statut et de la position des peintres de cour eux-mêmes comme l'atteste l'exemple des artistes de Marie-Thérèse. Ce passage du modèle des peintres de cour directement rattachés au souverain vers des artistes soumis à une formation toujours plus académique, qui institutionnalise l'enseignement et l'organisation des peintres pour les intérêts de l'État, se fait durant cette période où Meytens, le peintre de cour réputé, est en même temps directeur de l'Académie. Les portraits royaux sont réalisés pour la plupart d'entre eux par des peintres, même non viennois et non autrichiens, qui ont suivi l'enseignement de l'Académie viennoise tout en étant très souvent nommés Kammermaler.

Après 1770 et la longue période d'influence du peintre suédois Meytens, portraitiste de la souveraine et directeur de l'Académie, celle-ci est animée par de nouveaux artistes particulièrement actifs dans les années 1770, influencés par de nouveaux courants, tout particulièrement par le classicisme de Winckelmann et de son entourage. Après la mort de Meytens, le peintre Anton von Maron, prend ainsi la relève comme portraitiste de Marie-Thérèse. Disposant d'un nom et d'une réputation, il joue notamment un rôle important comme conseiller dans la réorganisation de l'Académie de Vienne.

Peut-on, pour autant, en déduire que tous les portraits de Marie-Thérèse sont uniquement structurés par la cour viennoise et par l'Académie de Vienne ? Et si oui de quelle manière ? Penchons-nous davantage sur ces peintres et en premier sur Meytens. 